\title{
Diagnostic and biological significance of microRNA-192 in pancreatic ductal adenocarcinoma
}

\author{
CHENYAN ZHAO $^{1,2^{*}}$, JING ZHANG $^{1 *}$, SHUHUI ZHANG ${ }^{1}$, DANGHUI YU $^{1}$, YING CHEN $^{1}$, \\ QINGHUA LIU ${ }^{1}, \mathrm{MIN} \mathrm{SHI}^{1}, \mathrm{CANRONG} \mathrm{NI}^{1}$ and MINGHUA ZHU ${ }^{1}$ \\ ${ }^{1}$ Department of Pathology, Changhai Hospital, Second Military Medical University, Shanghai; \\ ${ }^{2}$ Department of Pathology, Obstetrics and Gynecology Hospital of Fudan University, Shanghai, P.R. China
}

Received December 20, 2012; Accepted March 28, 2013

DOI: 10.3892/or.2013.2420

\begin{abstract}
Pancreatic ductal adenocarcinoma (PDAC) is one of the most lethal types of human cancer. In the present study, we evaluated serum microRNA-192 (miR-192) as a potential biomarker in patients with PDAC and investigated its biological functions in this disease. miRNA expression profiling of human PDACs and adjacent normal pancreatic tissues identified 16 upregulated miRNAs including miR-192 and 8 downregulated miRNAs. Quantitative real-time polymerase chain reaction (PCR) revealed elevation of serum miR-192 levels in PDAC patients relative to these levels in duodenal adenocarcinoma patients and healthy controls. Receiver operating characteristic analysis demonstrated that serum miR-192 had a sensitivity of $76 \%$ and a specificity of $55 \%$ for detecting PDAC. Ectopic expression of miR-192 in PANC-1 pancreatic cancer cells enhanced cell proliferation and migration, reduced apoptosis and promoted cell cycle progression from the G0/G1 to the S phase. Western blot analysis showed that enforced expression of miR-192 decreased the expression of smad-interacting protein 1 (SIP1) and altered a set of cell cycle-related genes in the PANC-1 cells. miR-192 overexpression increased tumor volume in an orthotopic pancreatic cancer mouse model, coupled with suppression of SIP1 and elevation of collagen I. In conclusion, serum miR-192 may serve as a sensitive diagnostic biomarker for PDAC. Overexpression of miR-192 contributes to tumor growth and progression in PDAC, which is associated with repression of SIP1 and alteration of cell cycle regulatory genes.
\end{abstract}

Correspondence to: Professor Minghua Zhu, Department of Pathology, Changhai Hospital, Second Military Medical University, 168 Changhai Road, Shanghai 200433, P.R. China

E-mail: mhzhu2000@hotmail.com; mhzhu07@126.com

*Contributed equally

Key words: pancreatic ductal adenocarcinoma, microRNA, diagnosis, growth, apoptosis

\section{Introduction}

Pancreatic ductal adenocarcinoma (PDAC) ranks among the most lethal types of human cancer, with a 5-year survival rate of lower than $5 \%$ and a median survival time of less than 6 months $(1,2)$. Although several non-invasive imaging techniques such as magnetic resonance imaging have been used to detect PDAC, early diagnosis of this disease is still clinically challenging as these methods do not reliably detect pancreatic tumors smaller than $1 \mathrm{~cm}$ in size (3). Consequently, in most cases, PDAC is not detected until at an advanced stage when potentially curative therapies are not available.

The identification of serum biomarkers has raised the possibility of developing an easy-to-perform and non-invasive approach to tumor diagnosis. Serum carbohydrate antigen 19-9 (CA 19-9) is the most well-established biomarker of pancreatic cancer, with a median sensitivity of $79 \%$ (range $70-90 \%$ ) and median specificity of $82 \%$ (range 68-91\%) (4). However, this marker has several major limitations, including lack of production in patients carrying the Lewis-negative genotype (5), frequent elevation in other malignancies and benign conditions, and ineffectiveness in detecting early stage cancers (6). Some alternative serum biomarkers such as tissue polypeptide antigen, macrophage inhibitory cytokine-1 and osteopontin have been explored (7). However, none of these potential biomarkers satisfy the requirements needed for the reliable diagnosis of PDACs.

Recent evidence indicates the potential value of serum microRNAs (miRNAs) in distinguishing malignant from benign lesions $(8,9)$. miRNAs are a large family of non-coding, single-stranded RNAs of 22 nucleotides, which negatively regulate gene expression by binding to the 3'-untranslated region (UTR) of target mRNAs, leading to accelerated mRNA turnover and/or translational repression (9). miRNAs are implicated in a wide range of biological processes including cell differentiation, proliferation, apoptosis and tumorigenesis (10). Microarray experiments revealed frequent and marked overexpression of miR-10a, miR-92 and miR-17-5p in pancreatic cancer cell lines (11). PDACs exhibit higher expression of miR-21, miR-221, miR-155, miR-100 and miR-181b than that in benign pancreatic lesions (12). miRNAs are highly stable in serum, plasma and other body fluids. This property, combined with altered expression in malignant diseases, 
makes circulating miRNAs potential non-invasive biomarkers for cancer detection.

The diagnostic value of circulating miRNAs has been described for pancreatic cancer. A panel of 7 miRNAs (i.e., miR-20a, miR-21, miR-24, miR-25, miR-99a, miR-185 and miR-191) was found to have high sensitivity and specificity for distinguishing pancreatic cancer from cancer-free controls and from chronic pancreatitis (13). Profiling the expression of miR-21, miR-210, miR-155 and miR-196a in plasma can differentiate pancreatic adenocarcinoma patients from healthy controls (14). To extend the list of circulating miRNA candidates for PDAC diagnosis, in the present study, we employed microarray techniques to profile and compare the expression of miRNAs in PDAC and normal pancreatic tissues. Additionally, the biological functions of representative miRNAs with markedly altered expression levels in PDAC were assessed, in order to provide a better understanding of their diagnostic relevance.

\section{Materials and methods}

Ethics. All procedures involving human subjects were approved by the Ethics Committee of the Secondary Military Medical University, Shanghai, China (no. CTcp008) in accordance with the Helsinki Declaration, and written informed consent was obtained from each patient enrolled. All animal experiments were undertaken in accordance with the National Institutes of Health Guide for the Care and Use of Laboratory Animals, with the approval of the Scientific Investigation Board of the Second Military Medical University (no. Lacp0010).

Patients and samples. We enrolled 80 consecutive PDAC patients (48 men and 32 women, aged 36-81 years) who underwent surgical resection during the period from January 2008 to June 2010 at the Department of Pancreatic Surgery of Changhai Hospital (Shanghai, China). All PDAC patients were pathologically diagnosed by biopsy, and the patients with prior anticancer treatments were excluded from this study. Resected tumor specimens and adjacent normal pancreatic tissues from 10 of this cohort of patients were collected for miRNA profiling analysis using microarray technology. Serum samples harvested from the remaining 70 PDAC patients, 17 age-matched duodenal adenocarcinoma patients, and 40 age-matched healthy controls were examined for expression of the miRNAs of interest using quantitative realtime polymerase chain reaction (qRT-PCR).

Pancreatic cancer cell lines and culture. Pancreatic cancer cell lines (sw1990, BxPC-3, MIA Paca-2, PANC-1, Capan-1, and AsPC-1) were purchased from the Institute of Cellular Research, Chinese Academy of Science, Shanghai, China. Cells were cultured in Dulbecco's minimum essential medium (Invitrogen Life Technologies, Grand Island, NY, USA) with $10 \%$ heat-inactivated fetal bovine serum (FBS), 100,000 U/1 penicillin and $100 \mathrm{mg} / \mathrm{l}$ streptomycin at $37^{\circ} \mathrm{C}$ in a $5 \% \mathrm{CO}_{2}$ incubator.

miRNA microarray analysis. miRNA expression profile analysis was performed using an Agilent miRNA microarray platform (Agilent Technologies Inc., Santa Clara, CA, USA) that consists of 713 probes and can simultaneously measure
713 miRNAs, as describe previously (15). Total RNA was extracted from human PDACs and adjacent normal pancreatic tissues using a mirVana ${ }^{\mathrm{TM}}$ PARIS kit (AM1561; Ambion, Austin, TX, USA) according to the manufacturer's instructions. The quality of the total RNA isolated was checked with a microcapillary electrophoresis system (Agilent 2100 Bioanlyzer). Cy3 labeling of RNA was performed using an miRNA labeling reagent and hybridization kit (Agilent 5190-0408; both from Agilent Technologies Inc., Palo Alto, CA, USA). The sample pre-treatment and probe hybridization were carried out according to the standard Agilent microarray protocol. A microarray scanner and Feature Extraction software (both from Agilent) were employed for microarray data collection and analysis. The raw microarray data were normalized using the variance stabilization and normalization method. Each sample had at least three duplicates on the microarray. A two-sample t-test was used for the statistical analysis, and a p-value $<0.05$ was considered to indicate a statistically significant result. In each group, the proportion of samples with a detection value of 1 was required to be higher than $60 \%$.

Locked nucleic acid-based in situ hybridization (LNA-ISH). LNA-ISH was performed using LNA $^{\mathrm{TM}}$ probes against miR-192 (Exiqon, Vedbaek, Denmark) on 5 frozen human PDAC samples according to the manufacturer's protocol, as described previously (16). Briefly, after prehybridization for 6-8 h, the prepared slides were incubated with a hybridization buffer containing a digoxigenin (DIG)-labeled LNA ${ }^{\mathrm{TM}}$ probe in a hybridization oven overnight at $53^{\circ} \mathrm{C}$. A negative parallel set was hybridized with non-miRNA probes as a measure of the probe specificity. After several washes, the slides were incubated with an anti-DIG Fab fragment $(1: 2,000)$ overnight in a humidified chamber at $4^{\circ} \mathrm{C}$. A colorimetric detection reaction was performed using NBT/BCIP ReadyMix (Roche, Mannheim, Germany) for $48 \mathrm{~h}$ at $4^{\circ} \mathrm{C}$, followed by counterstaining using methyl green (Trevigen Inc., Gaithersburg, MD, USA).

miRNA expression detected by $q R T-P C R$. Total RNA was extracted as described above, and the reverse transcription reaction was carried out using a TaqMan microRNA reverse transcription kit (Applied Biosystems, Foster City, CA, USA). Quantitative PCR was performed using a StepOnePlus ${ }^{\mathrm{TM}}$ RT-PCR kit (Applied Biosystems). Cycle threshold (Ct) values were calculated using SDS 1.4 software (Applied Biosystems). The relative expression of miRNAs was calculated with the $2^{-\Delta \Delta \mathrm{Ct}}$ method and normalized against U6 snRNA (17).

Gene transfection and establishment of stable cell clones. The miR-192 expression construct was constructed by the cloning of annealed oligonucleotides of mature miR-192 into the pGC-LV expression vector (GeneChem, Shanghai, China). A scrambled sequence without significant homology to rat, mouse or human gene sequences was used as a control. The miR-192 expression constructs were transfected into the PANC-1 cell line using Lipofectamine 2000 (Invitrogen Life Technologies, Carlsbad, CA, USA) according to the manufacturer's instructions. Real-time PCR was used to confirm the overexpression of miR-192. 
For stable expression of miR-192 in cells, a lentivirusbased expression system was employed. The oligonucleotides for pre-miR-192 expression were synthesized and inserted into the lentiviral expression vector carrying an enhanced green fluorescent protein (EGFP) tag (Clontech Laboratories, Mountain View, CA, USA). The miR-192-expressing plasmid or EGFP control plasmid was transfected into HEK 293T packaging cells using the Lentiphos ${ }^{\mathrm{TM}}$ HT packaging system. The recombinant lentiviral particles were generated and purified following the manufacturer's instructions. PANC-1 cells were grown to $70-80 \%$ confluence in 6-well plates and infected with recombinant lentivirus for $2 \mathrm{~h}$. After $48 \mathrm{~h}$, the infected cells were selected with fresh medium containing $5 \mu \mathrm{g} / \mathrm{ml}$ puromycin for 4-5 passages. Positively infected cells with EGFP expression were sorted by flow cytometry and cultured until implantation.

Cell proliferation and colony formation. Cell proliferation analysis and colony formation assay were performed as described previously (18). Transfected PANC-1 cells were plated in sextuplicate in 96-well microplates at a density of $1.5 \times 10^{3}$ cells/well. Cells were counted daily over a 7 -day period by the 3-(4,5-dimethylthiazol-2-yl)-2,5-diphenyltetrazolium bromide (MTT) assay using the CellTiter 96 Non-Radioactive Cell Proliferation Assay (Promega Corporation, Madison, WI, USA). The dark-blue crystals of MTT-formazan were dissolved by shaking the plates at room temperature for $10 \mathrm{~min}$, and the absorbance was then measured on a Bio-Rad microplate reader at a test wavelength of $490 \mathrm{~nm}$ and a reference wavelength of $630 \mathrm{~nm}$. Each growth curve showed the means and standard deviation (SD) of at least three independent experiments.

The colony formation assay was carried out as described previously (18). Briefly, transfected PANC-1 cells were plated in 6-well plates at a density of 1,000 cells/well. After 10 days, cells were washed with PBS, fixed in 10\% methanol for $15 \mathrm{~min}$, and stained in Giemsa for $20 \mathrm{~min}$. Colonies consisting of $>50$ cells were scored. Each experiment was repeated three times.

Cell cycle and apoptosis analysis. Cellular DNA content was analyzed by flow cytometry as described previously (18). Briefly, PANC-1 transfectants in a logarithmic growth phase were harvested, fixed in ethanol, and incubated with $0.5 \mathrm{mg} /$ $\mathrm{ml}$ of propidium iodide (PI) along with $0.1 \mathrm{mg} / \mathrm{ml}$ of RNase A (200 KU; Calbiochem, San Diego, CA, USA), prior to cell cycle analysis. Apoptosis was measured with an Annexin V/FITC kit (LHK601-100; Bender Medsystems, Austria) according to the manufacturer's protocol. Each assay was repeated three times.

In vitro wound healing assay. Cell migration determined by the scratch assay was performed as described previously (19). Briefly, an open area or 'scratch' was produced in 90\% PANC-1 cell monolayers using a $200-\mathrm{ml}$ pipette tip. The cell cultures were washed for several times to remove the displaced cells in the open area, and then maintained in McCoy's 5A culture medium for $48 \mathrm{~h}$. The extent of wound closure was examined by phase-contrast microscopy.

Tumorigenicity in nude mice. The tumorigenicity assay was performed as previously described (18). Nude mice were purchased from the Experimental Animal Center of Shanghai
(Shanghai, China) and randomly divided into 2 groups $(\mathrm{n}=4)$. To establish orthotopic pancreatic tumors, animals were anesthetized and the pancreas was exposed via an abdominal incision. The mice were injected into the pancreas at a single site with $2 \times 10^{6}$ miR-192-overexpressing or empty vectortransduced PANC-1 cells. After tumor cell implantation, the pancreas was carefully returned to the peritoneal cavity and the abdomen was closed. All animals were monitored for 30 days for tumor formation and then sacrificed. The mean tumor volume was calculated.

Protein extraction and western blot analysis. In the present study, we used primary antibodies against S-phase kinaseassociated protein 2 (SKP-2; ab68455; 1:100), P27 (ab7961; 1:100), cyclin D1 (ab6152; 1:100), cyclin D2 (ab3087; 1:100), P130 (ab80628; 1:500), P107 (ab2451; 1:500), CDC2 (ab18; 1:500), cyclin-dependent kinase 4 (CDK4; ab7955; 1:500), smad interacting protein 1 (SIP1; ab25837; 1:2,000), collagen I (ab34710; 1:5,000) (all from Abcam, Cambridge, UK), and P21 (sc-6246; 1:50), retinoblastoma protein (RB1; sc-73598; 1:500), and $\beta$-actin; $(1: 2,000)$ (all from Santa Cruz Biotechnology, Inc., Santa Cruz, CA, USA). Protein isolation and western blotting were carried out as previously described (18). Briefly, tissues were lysed in a $10 \mathrm{mM}$ Tris-buffer ( $\mathrm{pH}$ 7.4) containing $1 \%$ sodium dodecyl sulfate (SDS) and complete protease inhibitors (Roche). Samples of the lysates $(50 \mu \mathrm{g})$ were separated by SDS-polyacrylamide gel electrophoresis $(12 \%$ polyacrylamide gel), transferred to polyvinylidene fluoride membranes (Millipore, Bedford, MA, USA), and probed with the primary antibodies. After incubation with the horseradish peroxidaseconjugated secondary antibody (Santa Cruz Biotechnology), the blots were visualized with enhanced chemiluminescence detection reagents (sc-2048, Santa Cruz Biotechnologies). The intensity of each band was measured using a Fluor-S MultiImager and Quantity-One software (Bio-Rad, Hercules, CA, USA).

Statistical analysis. Data are presented as means \pm SD. All statistical calculations were carried out using SPSS.11 software (SPSS, Chicago, IL, USA). Significant differences between two groups were determined using the Student's t-test. The difference among the means of multiple groups was analyzed by one-way analysis of variance followed by the Tukey's test. Receiver operating characteristic (ROC) curves were established to evaluate the diagnostic potential of miR-192. The relationship between miR-192 expression and clinicopathological features was analyzed using the Chi-square test. A difference was defined as significant at $\mathrm{p}<0.05$.

\section{Results}

Distinct miRNA expression profiles in PDAC and normal pancreatic tissues. The differentially expressed miRNAs in pooled tissue extracts of 10 PDACs and adjacent normal controls were identified. Eight miRNAs (miR-194*, miR-192*, miR-602, miR-801, miR-194, miR-192, miR-212 and miR-34a*) showed upregulated expression and 8 (miR-923, miR-139-3p, miR-513a-5p, miR-630, miR-30c-1*, miR-887, miR-508-5p, and miR-516a-5p) showed downregulated expression in PDAC pools, with a cut-off value of a 1.15 -fold change (Table I). Since 
Table I. Differentially expressed miRNAs in PDAC and normal pancreatic tissues.

\begin{tabular}{|c|c|c|c|c|}
\hline miRNA & Mean CA & Mean N & Fold (CA/N) & P-value \\
\hline hsa-miR-194* & 2.037867 & 0.854847 & 2.383897 & 0.016501 \\
\hline hsa-miR-192* & 4.006635 & 2.186735 & 1.832245 & 0.021953 \\
\hline hsa-miR-602 & 3.635497 & 2.793224 & 1.301542 & 0.019506 \\
\hline hsa-miR-801 & 6.470025 & 5.068935 & 1.276407 & 0.03212 \\
\hline hsa-miR-194 & 9.243658 & 7.474524 & 1.236689 & 0.010702 \\
\hline hsa-miR-192 & 9.850274 & 8.345187 & 1.180354 & 0.024704 \\
\hline hsa-miR-212 & 5.119245 & 4.390118 & 1.166084 & 0.009916 \\
\hline hsa-miR-34a* & 3.098899 & 2.69141 & 1.151404 & 0.022457 \\
\hline hsa-miR-923 & 13.0211 & 14.90239 & 0.873759 & 0.00042 \\
\hline hsa-miR-139-3p & 3.817374 & 4.661482 & 0.818918 & 0.030804 \\
\hline hsa-miR-513a-5p & 4.087004 & 5.163924 & 0.791453 & 0.025165 \\
\hline hsa-miR-630 & 6.331195 & 8.517886 & 0.743282 & 0.024383 \\
\hline hsa-miR-30c-1* & 1.7912 & 2.42261 & 0.739368 & 0.044192 \\
\hline hsa-miR-887 & 2.955096 & 4.281118 & 0.690263 & 0.04656 \\
\hline hsa-miR-508-5p & -0.07768 & 1.467253 & 0.681546 & 0.001305 \\
\hline hsa-miR-516a-5p & 1.397406 & 3.67883 & 0.379851 & 0.015698 \\
\hline
\end{tabular}

miRNA, microRNA; PDAC, pancreatic ductal adenocarcinoma. CA, cancer tissues; $\mathrm{N}$, normal pancreatic tissues.
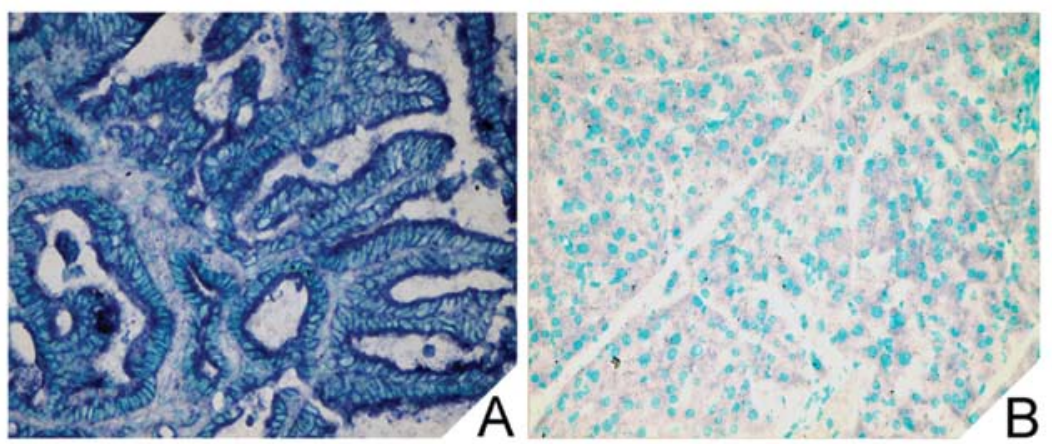

Figure 1. Tissue miR-192 expression as detected by LNA-ISH. Positive cytoplasmic staining for miR-192 was noted in (A) human PDAC tissues but not in (B) adjacent normal pancreatic tissues. Original magnification, $\mathrm{x} 400$.

miR-192 is linked to pancreatic cancer progression (10) and its function has been studied to a lesser extent in this malignancy, in this study we focused on its expression, biological role, and diagnostic potential in PDAC.

To confirm the microarray results, tissue miR-192 expression was examined by LNA-ISH. Positive cytoplasmic staining for miR-192 was evident in tumor cells but was rarely noted in the stromal cells of the PDAC specimens (Fig. 1A). However, no miR-192 positivity was found in the adjacent normal pancreatic tissues (Fig. 1B).

Clinical significance of circulating miR-192 in PDAC. Notably, PDAC patients had $>5$-fold greater concentrations of serum miR-192 than duodenal adenocarcinoma patients or healthy controls ( $\mathrm{p}<0.05$ for each comparison) (Fig. 2A). However, no significant difference in the serum miR-192 level was observed between duodenal adenocarcinoma patients and healthy controls.
To further assess the diagnostic significance of serum miR-192 levels in differentiating between PDAC patients and healthy individuals, ROC curves were constructed (Fig. 2B). The area under the curve (AUC) was 0.63 (95\% CI, 0.51-0.75). Using an optimal cut-off point, the sensitivity was $76 \%$ and the specificity was 55\%. Table II shows the association of the serum level of miR-192 and the clinicopathological factors of the 70 PDAC patients. No correlation was found between the serum miR-192 level and any variable tested, including gender, age, tumor size, or pathological type $(\mathrm{p}>0.05)$.

Effects of miR-192 overexpression on cell growth, survival, and migration of pancreatic cancer cells. miR-192 was the most abundant in AsPC-1 cells, followed by Capan-1, SW1990, BxPC-3, PANC-1 and MIA Paca-2 cells (Fig. 3A). Similar findings were noted in their culture supernatants (Fig. 3B). The delivery of miR-192-expressing constructs (pGC-LV-miR-192) resulted in a significant elevation in miR-192 expression, as 
Table II. Correlation of serum miRNA-192 levels with clinicopathological parameters of the PDAC patients.

\begin{tabular}{|c|c|c|c|}
\hline \multirow[b]{2}{*}{ Characteristic } & \multirow[b]{2}{*}{$\mathrm{N}$} & \multicolumn{2}{|c|}{ miR-192 } \\
\hline & & Value & P-value \\
\hline \multicolumn{4}{|l|}{ Gender } \\
\hline Male & 42 & $0.19 \pm 0.22$ & 0.838 \\
\hline Female & 28 & $0.20 \pm 0.18$ & \\
\hline \multicolumn{4}{|l|}{ Age (years) } \\
\hline$\leq 60$ & 32 & $0.19 \pm 0.22$ & 0.975 \\
\hline$>60$ & 38 & $0.19 \pm 0.18$ & \\
\hline \multicolumn{4}{|c|}{ Location of tumor ${ }^{\mathrm{a}}$} \\
\hline Head & 40 & $0.20 \pm 0.19$ & 0.858 \\
\hline Body/Tail & 26 & $0.19 \pm 0.22$ & \\
\hline \multicolumn{4}{|c|}{ Size of tumor $(\mathrm{cm})^{\mathrm{a}}$} \\
\hline$\leq 2.0$ & 8 & $0.19 \pm 0.24$ & 0.945 \\
\hline$>2.0$ & 58 & $0.20 \pm 0.20$ & \\
\hline \multicolumn{4}{|c|}{ Histological grade ${ }^{\mathrm{a}}$} \\
\hline G1 & 2 & $0.14 \pm 0.11$ & 0.325 \\
\hline $\mathrm{G} 2$ & 50 & $0.18 \pm 0.23$ & \\
\hline G3 & 14 & $0.29 \pm 0.18$ & \\
\hline \multicolumn{4}{|l|}{$\mathrm{PT}^{\mathrm{a}}$} \\
\hline $\mathrm{T} 1$ & & & 0.075 \\
\hline $\mathrm{T} 2$ & 26 & $0.16 \pm 0.19$ & \\
\hline $\mathrm{T} 3$ & 26 & $0.18 \pm 0.20$ & \\
\hline $\mathrm{T} 4$ & 14 & $0.40 \pm 0.27$ & \\
\hline \multicolumn{4}{|l|}{$\mathrm{PN}^{\mathrm{a}}$} \\
\hline Absent & 39 & $0.17 \pm 0.22$ & 0.952 \\
\hline Present & 27 & $0.19 \pm 0.18$ & \\
\hline \multicolumn{4}{|l|}{ PTNM $^{a}$} \\
\hline I & 15 & $0.14 \pm 0.21$ & 0.195 \\
\hline II & 21 & $0.26 \pm 0.19$ & \\
\hline III & 27 & $0.20 \pm 0.20$ & \\
\hline IV & 3 & $0.30 \pm 0.48$ & \\
\hline \multicolumn{4}{|l|}{ CA19-9 $9^{\mathrm{a}}$} \\
\hline$\leq 37$ & 18 & $0.15 \pm 0.20$ & 0.116 \\
\hline$>37$ & 48 & $0.22 \pm 0.2$ & \\
\hline
\end{tabular}

aSome records were missing due to incomplete case records. miRNA, microRNA; PDAC, pancreatic ductal adenocarcinoma; PN, pathological lymph node; PT, pathological tumor; PTNM, pathological tumor lymph node and metastasis.

compared to the control cells transfected with the pGC-LV empty vector ( $<<0.01$; Fig. 3C).

The MTT assay revealed that miR-192 overexpression significantly promoted cell proliferation in PANC-1 cells in comparison with that in the empty vector-transfected cells $(\mathrm{p}<0.01 ;$ Fig. 3D). The colony formation assay showed that miR-192-overexpressing PANC-1 cells had a significantly higher colony formation rate than the empty vector transfectants $(47.5 \pm 2.3$ vs. $27.3 \pm 2.0 \%$ ) (Fig. $4 \mathrm{~A})$. Overexpression
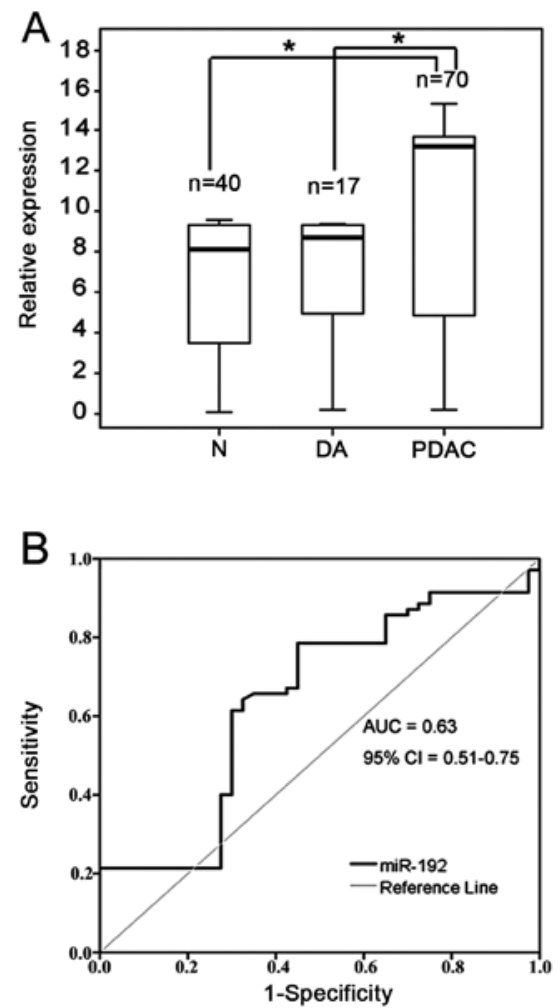

Figure 2. Evaluation of the diagnostic significance of serum miR-192 in PDAC. (A) Quantitative real-time PCR analysis of serum miR-192 levels in PDAC patients, duodenal adenocarcinoma (DA) patients, and normal controls $(\mathrm{N})$. The thick horizontal line indicates the mean $(\mathrm{p}<0.05)$. (B) ROC curve analysis for discrimination between 70 PDAC patients and 40 healthy controls according to serum miR-192 level.

of miR-192 increased the percentage of cells in the S phase and decreased the proportion of cells in the G0/G1 and G2/M phases (Fig. 4B). Moreover, there was a significantly lower apoptotic index in the miR-192-overexpressing cells than that in the control transfectants $(4.1 \pm 0.9$ vs. $7.2 \pm 1.1 \%)(\mathrm{p}<0.01$; Fig. 4C). In vitro wound healing assay further showed that enforced miR-192 expression potentiated the migration of PANC-1 cells, as evidenced by acceleration of wound closure (Fig. 4D).

Alteration of cell cycle-related genes by miR-192 overexpression. Western blot analysis revealed that the expression levels of P21, P27, P107, P130 and RB were lower in miR192-overexpressing PANC-1 cells than that in the empty vector-transfected counterparts (Fig. 5). In contrast, there were increased protein levels of cyclin D1, cyclin D2, CDK4, SKP-2 and CDC2 in the miR-192-overexpressing PANC-1 cells relative to the control transfectants (Fig. 5).

Effects of miR-192 overexpression on tumor growth in a PANC-1 xenograft model. miR-192-overexpressing PANC-1 cells formed significantly larger tumors in nude mice when compared with those in the control transfectants $(0.47 \pm 0.24$ vs. $0.02 \pm 0.01 \mathrm{~cm}^{3}, \mathrm{p}<0.01$ ) (Fig. $6 \mathrm{~A}$ ). Immunohistochemistry revealed that miR-192 xenograft tumors displayed stronger immunoreactivity for collagen I when compared with that in the control xenograft tumors (Fig. 6B). Western blot analysis 
A

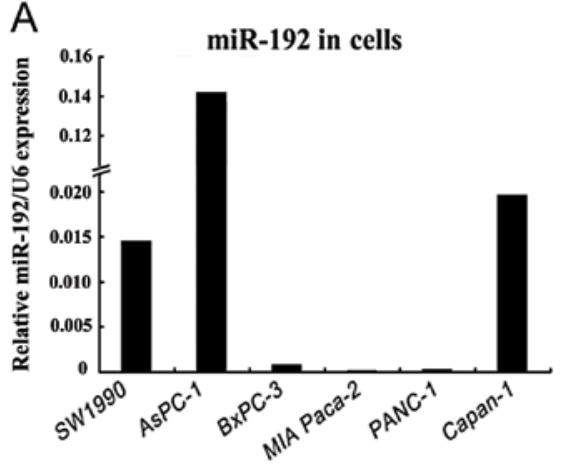

C

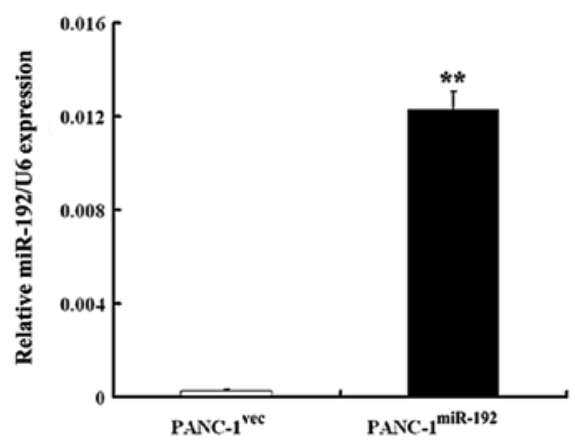

B

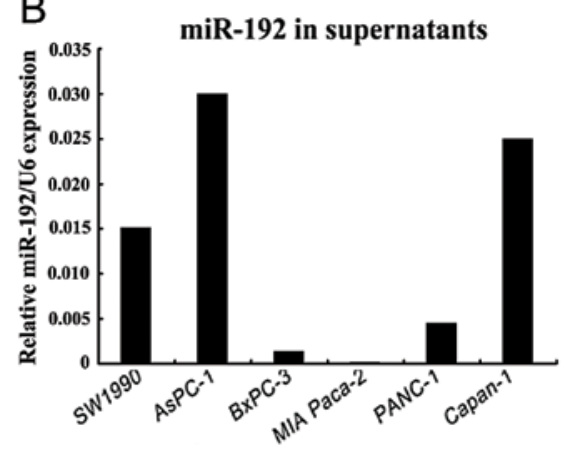

D

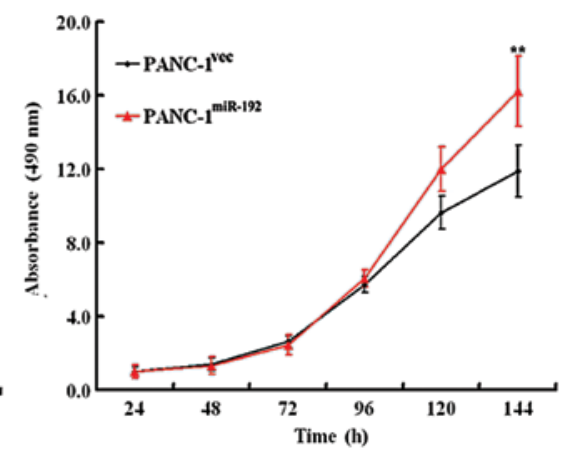

Figure 3. Effects of miR-192 overexpression on the proliferation of pancreatic cancer cells. (A and B) Quantitative real-time PCR analysis of miR-192 levels in a panel of human pancreatic cancer lines and their culture supernatants. (C) The relative expression of miR-192 in miR-192-overexpressing and empty vector-transfected PANC-1 cells. " $\mathrm{p}<0.01$ between the two groups. (D) Evaluation of cell proliferation using the MTT assay. Growth curves were plotted as optical density $(490 \mathrm{~nm})$ vs. time. " $\mathrm{p}<0.01$ between the two groups.

A

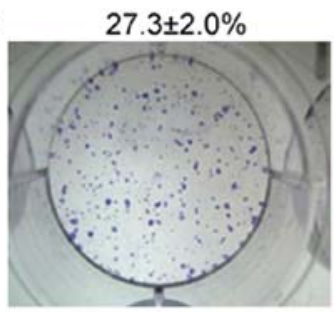

PANC-1 ${ }^{\text {vec }}$

B

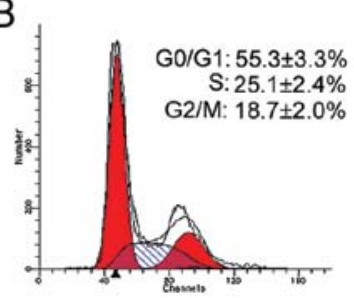

PANC-1 ${ }^{\text {vec }}$

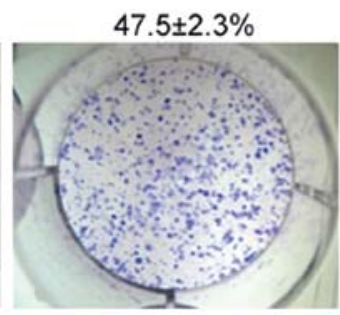

PANC-1 ${ }^{\text {miR-192 }}$

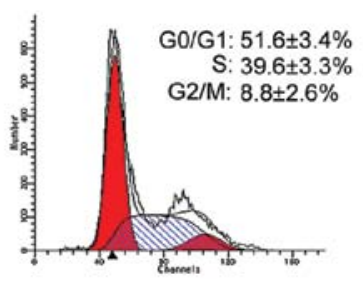

PANC-1 ${ }^{\text {miR-192 }}$

C

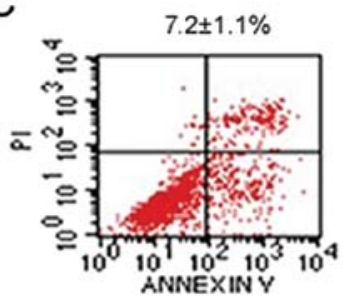

PANC-1 ${ }^{\text {vec }}$

D

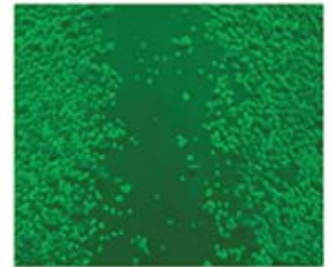

PANC-1 ${ }^{\text {vec }}$

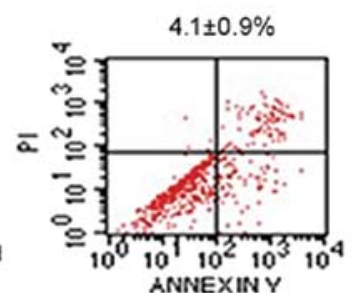

PANC-1 ${ }^{\text {miR-192 }}$

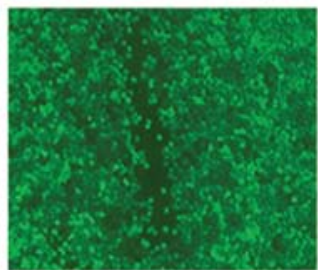

PANC-1 ${ }^{\text {miR-192 }}$

Figure 4. Effects of miR-192 overexpression on colony formation, cell cycle progression, apoptosis, and migration in PANC-1 cells. (A) Colony formation assay revealed a significantly higher colony formation rate for miR-192-overexpressing PANC-1 cells. (B) Cell cycle distribution in miR-192-overexpressing and empty vector-transfected PANC-1 cells stained by PI. (C) Apoptosis analysis using Annexin V and PI staining. miR-192-overexpressing cells had a significantly lower apoptotic index than empty vector-transfected cells. (D) Cell migration assessed by in vitro wound healing assay. Original magnification, $\mathrm{x} 40$.

further confirmed the immunohistochemical results (Fig. 6C). In contrast, the SIP1 protein level was profoundly reduced in the miR-192 xenograft tumors when compared to that in the control tumors (Fig. 6C). Additionally, miR-192-overexpressing PANC-1 cells showed reduced SIP1 protein levels and elevated collagen I protein levels (Fig. 6C). 


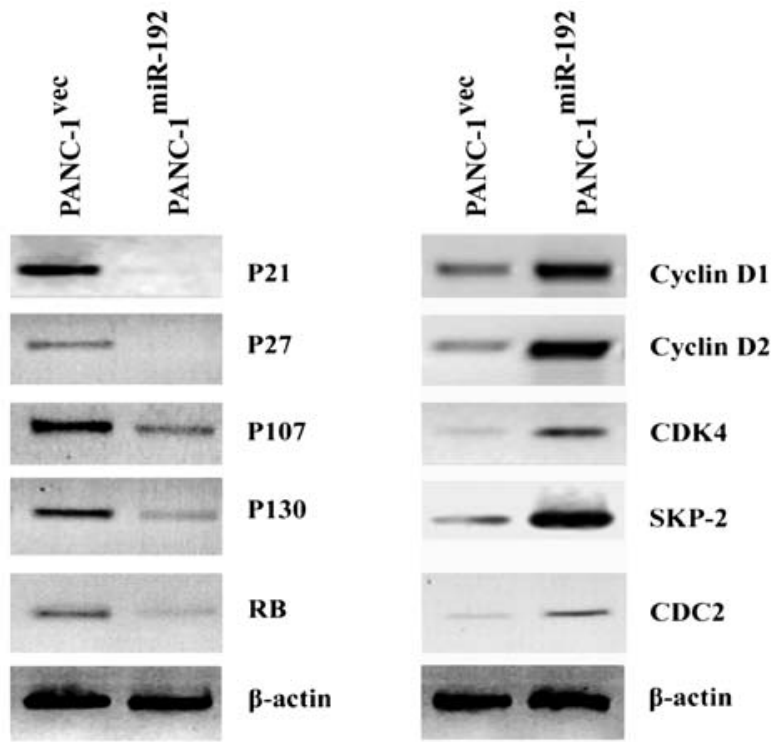

Figure 5. Western blot analysis of the protein levels of cell cycle-related genes in empty vector-transfected and miR-192-overexpressing PANC-1 cells.

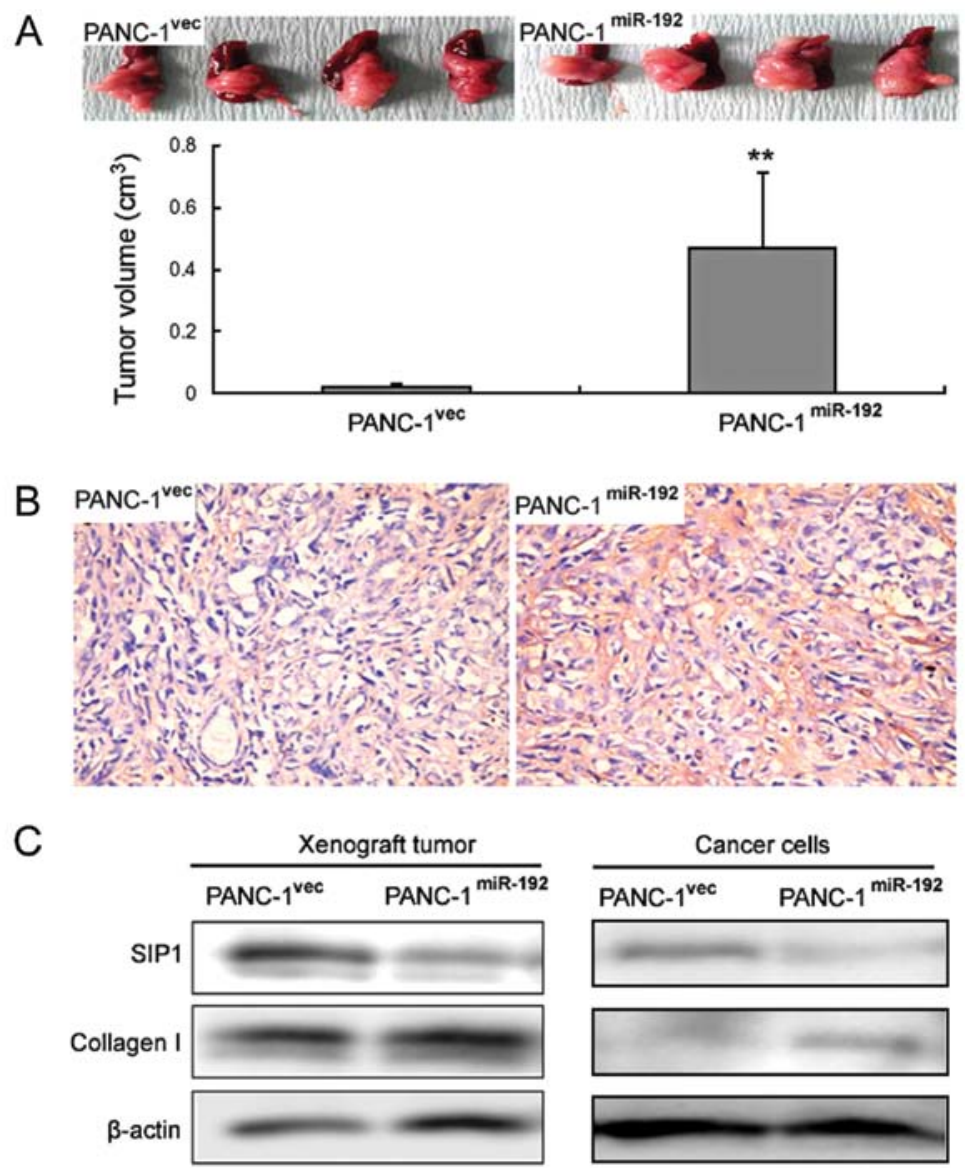

Figure 6. Effects of miR-192 overexpression on tumor growth in a PANC-1 xenograft model. (A) Macroscopic images of xenograft tumors from miR-192-overexpressing and empty vector-transduced PANC-1 cells. (B) Immunohistochemical staining for collagen I in miR-192-overexpressing xenograft tumors and control tumors. Original magnification, x400. (C) Western blot analysis of SIP1 and collagen I protein levels in miR-192-overexpressing and control xenograft tumors.

\section{Discussion}

Serum miRNA profiles change during tumor develop- ment (11). Serum levels of miR-141 (an miRNA abundant in prostate cancer) can distinguish prostate cancer patients from healthy controls (12). Using the miRNA microarray 
technology, we identified a set of differentially expressed miRNAs, including miR-194 and miR-192, between human PDAC and adjacent normal pancreatic tissues. It has been shown that upregulation of miR-194 correlates with reduced expression of tumor-suppressor EP300 in metastatic PDAC cells (13), suggesting a favorable role of miR-194 in PDAC progression. In the present study, we focused on the less studied miR-192, attempting to determine its diagnostic and functional significance in PDAC. Our present data revealed that serum miR-192 concentrations were significantly higher in PDAC patients than these concentrations in duodenal adenocarcinoma patients and healthy controls. ROC curve analysis revealed that serum miR-192 levels were able to differentiate between PDAC patients and healthy individuals, with a sensitivity and specificity of 76 and 55\%, respectively. Although the discriminating power of miR-192 alone indicated by the AUC value (0.627) was relatively lower than the expectations of clinical utility, its combination with other miRNAs or protein biomarkers would improve predictive accuracy and robustness. Indeed, the combination of the four miRNAs (miR-21, miR-210, miR-155 and miR-196a) increased the AUC value from 0.62 to 0.69 ranging up to 0.82 for pancreatic cancer detection (14).

Cellular miRNAs can be encapsulated in exosome-like vesicles and released into the extracellular environment $(15,16)$. In support of this view, our data revealed that miR-192 was present in the culture supernatants of a panel of pancreatic cancer cell lines. The mechanism of miRNA release is still not completely understood. However, recent studies suggest that miRNA release may be mediated through a ceramidedependent secretory machinery (17). Our data indicated an upregulation of miR-192 in PDAC specimens compared to adjacent normal tissues. Moreover, serum miR-192 levels were elevated in PDAC patients relative to duodenal adenocarcinoma patients and healthy controls. These findings suggest that PDAC cells rather than non-malignant pancreatic cells abundantly express and release miR-192, which provide a rationale for the evaluation of circulating miR-192 as a potential biomarker for PDAC diagnosis.

Extensive studies indicate that miRNAs play critical roles in cancer development and progression. miR-192 can function as an effector and regulator of p53 and induce cell cycle arrest in tumor cells (18). Song et al (18) also found that miR-192 overexpression suppresses cell proliferation in colon cancer cell lines through induction of p53-dependent cell cycle arrest at both the $\mathrm{G} 1$ and $\mathrm{G} 2$ phases. In lung cancer cells, miR-192 was found to inhibit cell proliferation through targeting RB1 (19). Our results are highly inconsistent with these previous studies. We found that ectopic expression of miR-192 promoted cell proliferation and facilitated cell cycle progression through the $\mathrm{G} 1$ to $\mathrm{S}$ phase in pancreatic cancer cell lines. Western blot analysis further revealed the deregulation of numerous cell cycle-related molecules. Cyclins and CDKs are important positive regulators with a central role in cell cycle progression. In contrast, a class of molecules called CDK inhibitors including P21 and P27 negatively regulates the cell cycle (20). The RB family members (RB, p107 and p130) are another group of negative regulators of the cell cycle and each of them can elicit G1-S growth arrest through interfering with E2F-dependent transcription (21). SKP-2, through its F-box domain, forms the SCF complex with SKP-1 and Cullin-1 to constitute an E3 ligase for ubiquitylation. SKP-2 has been found to promote $\mathrm{S}$-phase progression by degrading negative cell cycle regulators including P21, P27, P57 and P130 (22). Interestingly, we found that miR-192 overexpression diminished the expression of P21, P27, P107, P130 and RB and increased the expression of cyclin D1, cyclin D2, CDK4, CDC2 and SKP-2. These results provide an explanation at the molecular level for the promotion of PDAC cell proliferation by miR-192. The growth-promoting effects of miR-192 overexpression were also observed in the in vitro colony formation assay and in vivo xenograft tumor model. This inconsistency with previous reports $(18,19,23)$ may reflect the effects on the functional consequences of manipulating miRNA. Similar to miR-192, miR-17-5p is able to act as both an oncogene and a tumor suppressor in different types of cancer cells (24). In addition to promotion of cell growth, we noted that enforced expression of miR-192 attenuated cell apoptosis and stimulated cell migration in pancreatic cancer cells. These findings collectively suggest that miR-192 acts as an oncogene in pancreatic cancer, thus representing a potential therapy target for this disease.

A single miRNA can coordinate a large number of target genes (25). Several miR-192 targets have been identified in different cells and organs. Microarray experiments have shown that 62 genes are downregulated following miR-192 overexpression, 8 of which have been validated as targets of miR-192 involved in cell cycle control (26). RB1 was found to be a target of miR-192, which mediates an effect on cell apoptosis through the caspase pathway (19). miR-192 inhibits nucleotide excision repair by targeting ERCC3 and ERCC4 in HepG2.2.15 cells (27). Our present data suggest SIP1 as a potential target of miR-192, as overexpression of miR-192 led to a marked repression of SIP1 protein expression in PANC-1 cells. In agreement with our data, a previous study demonstrated that miR-192 decreased endogenous SIP1 expression as well as reporter activity of an SIP1 3' UTR-containing luciferase construct in mouse mesangial cells (28). SIP1 was found to induce G1 phase cell cycle arrest in squamous carcinoma cells by repressing cyclin D1 transcription (29). In human RT112 bladder carcinoma cells, SIP1 also profoundly repressed the G1 to $\mathrm{S}$ phase transition (30). It remains to be determined, however, whether, and to what extent, downregulation of SIP1 mediates the growth-promoting effects of miR-192 in PDAC. In contrary to SIP1, we found an upregulation of collagen I protein by miR-192 overexpression. It has been documented that elevated collagen I synthesis facilitates pancreatic cancer spread by increasing cell proliferation, resistance to apoptosis, and migratory activity $(31,32)$. Imamichi et al (33) reported that collagen I promotes downregulation of E-cadherin expression in PANC-1 cells, which correlates with enhanced cell migration and invasiveness. The induction of collagen I expression may partially explain the favorable effects of miR-192 overexpression on PDAC cell survival and migration.

However, the various limitations of this study should be noted. The sample size was relatively small. Moreover, there was no information concerning the discriminating capacity of serum miR-192 in PDACs at different stages. The exact molecular mechanisms underlying the effects of miR-192 on pancreatic cancer cells await further elucidation. Identification of the direct, bona fide target gene of miR-192 will be the key 
to address this issue. Additionally, loss-of-function studies are needed to complement the results of gain-of-function studies and to further confirm the oncogenic role of miR-192 in PDAC.

In summary, our results demonstrate the potential of serum miR-192 in differentiating between PDAC patients and healthy controls. Enforced expression of miR-192 enhances proliferation and migration and reduces apoptosis in PDAC, which may be associated with alteration of cell cycle regulatory genes, downregulation of SIP1, as well as upregulation of collagen I. These findings propose miR-192 as a promising diagnostic and therapeutic target for PDAC.

\section{Acknowledgements}

This study was supported by the National Key Project of Scientific and Technical Supporting Programs of China (no. 2006BAI02A14) and National Natural Science Foundation of China (no. 30770996 and no. 81172310) to Minghua Zhu.

\section{References}

1. Jemal A, Siegel R, Xu J and Ward E: Cancer statistics, 2010. CA Cancer J Clin 60: 277-300, 2010.

2. Li D, Xie K, Wolff R and Abbruzzese JL: Pancreatic cancer. Lancet 363: 1049-1057, 2004.

3. Brand RE and Matamoros A: Imaging techniques in the evaluation of adenocarcinoma of the pancreas. Dig Dis 16: 242-252, 1998 .

4. Goonetilleke KS and Siriwardena AK: Systematic review of carbohydrate antigen (CA 19-9) as a biochemical marker in the diagnosis of pancreatic cancer. Eur J Surg Oncol 33: 266-270, 2007.

5. Kawai S, Suzuki K, Nishio K, et al: Smoking and serum CA19-9 levels according to Lewis and secretor genotypes. Int J Cancer 123: 2880-2884, 2008.

6. Koopmann J, Rosenzweig CN, Zhang Z, et al: Serum markers in patients with resectable pancreatic adenocarcinoma: macrophage inhibitory cytokine 1 versus CA19-9. Clin Cancer Res 12 442-446, 2006.

7. Bünger S, Laubert T, Roblick UJ and Habermann JK: Serum biomarkers for improved diagnostic of pancreatic cancer: a current overview. J Cancer Res Clin Oncol 137: 375-389, 2011.

8. Cortez MA, Welsh JW and Calin GA: Circulating microRNAs as noninvasive biomarkers in breast cancer. Recent Results Cancer Res 195: 151-161, 2012.

9. Redova M, Poprach A, Nekvindova J, et al: Circulating miR-378 and $\mathrm{miR}-451$ in serum are potential biomarkers for renal cell carcinoma. J Transl Med 10: 55, 2012.

10. Jung DE, Wen J, Oh T and Song SY: Differentially expressed microRNAs in pancreatic cancer stem cells. Pancreas 40 1180-1187, 2011.

11. Keller A, Leidinger P, Gislefoss R, et al: Stable serum miRNA profiles as potential tool for non-invasive lung cancer diagnosis. RNA Biol 8: 506-516, 2011.

12. Mitchell PS, Parkin RK, Kroh EM, et al: Circulating microRNAs as stable blood-based markers for cancer detection. Proc Natl Acad Sci USA 105: 10513-10518, 2008.

13. Mees ST, Mardin WA, Wendel C, et al: EP300 - a miRNAregulated metastasis suppressor gene in ductal adenocarcinomas of the pancreas. Int J Cancer 126: 114-124, 2010.
14. Wang J, Chen J, Chang $\mathrm{P}$, et al: MicroRNAs in plasma of pancreatic ductal adenocarcinoma patients as novel blood-based biomarkers of disease. Cancer Prev Res 2: 807-813, 2009.

15. Valadi H, Ekström K, Bossios A, Sjöstrand M, Lee JJ and Lötvall JO: Exosome-mediated transfer of mRNAs and microRNAs is a novel mechanism of genetic exchange between cells. Nat Cell Biol 9: 654-659, 2007.

16. Rabinowits G, Gercel-Taylor C, Day JM, Taylor DD and Kloecker GH: Exosomal microRNA: a diagnostic marker for lung cancer. Clin Lung Cancer 10: 42-46, 2009.

17. Kosaka N, Iguchi H, Yoshioka Y, Takeshita F, Matsuki Y and Ochiya T: Secretory mechanisms and intercellular transfer of microRNAs in living cells. J Biol Chem 285: 17442-17452, 2010.

18. Song B, Wang Y, Kudo K, Gavin EJ, Xi Y and Ju J: miR-192 regulates dihydrofolate reductase and cellular proliferation through the p53-microRNA circuit. Clin Cancer Res 14: 8080-8086, 2008.

19. Feng S, Cong S, Zhang X, et al: MicroRNA-192 targeting retinoblastoma 1 inhibits cell proliferation and induces cell apoptosis in lung cancer cells. Nucleic Acids Res 39: 6669-6678, 2011.

20. Vermeulen K, Van Bockstaele DR and Berneman ZN: The cell cycle: a review of regulation, deregulation and therapeutic targets in cancer. Cell Prolif 36: 131-149, 2003.

21. Classon M, Salama S, Gorka C, Mulloy R, Braun P and Harlow E: Combinatorial roles for pRB, p107, and p130 in E2F-mediated cell cycle control. Proc Natl Acad Sci USA 97: 10820-10825, 2000.

22. Frescas D and Pagano M: Deregulated proteolysis by the F-box proteins SKP2 and beta-TrCP: tipping the scales of cancer. Nat Rev Cancer 8: 438-449, 2008.

23. Braun CJ, Zhang X, Savelyeva I, et al: p53-Responsive microRNAs 192 and 215 are capable of inducing cell cycle arrest. Cancer Res 68: 10094-10104, 2008.

24. Cloonan N, Brown MK, Steptoe AL, et al: The miR-17-5p microRNA is a key regulator of the G1/S phase cell cycle transition. Genome Biol 9: R127, 2008.

25. Lim LP, Lau NC, Garrett-Engele P, et al: Microarray analysis shows that some microRNAs downregulate large numbers of target mRNAs. Nature 433: 769-773, 2005.

26. Georges SA, Biery MC, Kim SY, et al: Coordinated regulation of cell cycle transcripts by p53-inducible microRNAs, miR-192 and miR-215. Cancer Res 68: 10105-10112, 2008.

27. Zhang S, Hao J, Xie F, et al: Downregulation of miR-132 by promoter methylation contributes to pancreatic cancer development. Carcinogenesis 32: 1183-1189, 2011.

28. Katoh $\mathrm{M}$ and Katoh M: Integrative genomic analyses of ZEB2: Transcriptional regulation of ZEB2 based on SMADs, ETS1, HIF1 $\alpha$, POU/OCT, and NF- $\kappa$ B. Int J Oncol 34: 1737-1742, 2009.

29. Mejlvang J, Kriajevska M, Vandewalle C, et al: Direct repression of cyclin D1 by SIP1 attenuates cell cycle progression in cells undergoing an epithelial mesenchymal transition. Mol Biol Cell 18: 4615-4624, 2007.

30. Sayan AE, Griffiths TR, Pal R, et al: SIP1 protein protects cells from DNA damage-induced apoptosis and has independent prognostic value in bladder cancer. Proc Natl Acad Sci USA 106: 14884-14889, 2009.

31. Grzesiak JJ and Bouvet M: The alpha2beta1 integrin mediates the malignant phenotype on type I collagen in pancreatic cancer cell lines. Br J Cancer 94: 1311-1319, 2006.

32. Menke A, Philippi C, Vogelmann R, et al: Down-regulation of E-cadherin gene expression by collagen type I and type III in pancreatic cancer cell lines. Cancer Res 61: 3508-3517, 2001.

33. Imamichi $Y$, König A, Gress $T$ and Menke A: Collagen type I-induced Smad-interacting protein 1 expression downregulates E-cadherin in pancreatic cancer. Oncogene 26: 2381-2385, 2007. 